\title{
CARBapenem utilizatiON evaluation in a large community hospital (CARBON): A Quality Improvement Study
}

\author{
Jesse Janssen, Angus Kinkade, and Damen Man
}

\section{INTRODUCTION}

$\longrightarrow f$

arbapenems constitute a class of broad-spectrum antibiotics for the treatment of documented multidrug-resistant organisms and for use as empiric therapy in severe, life-threatening conditions, ${ }^{1,2}$ Hospital-acquired infections due to organisms such as Clostridium difficile and multidrug-resistant Pseudomonas aeruginosa are responsible for significant morbidity and mortality in the institutional health care setting. ${ }^{3}$ Increased use of carbapenems has been linked with a rising incidence of carbapenem-resistant Enterobacteriaceae, $C$. difficile, and other multidrug-resistant organisms. ${ }^{4}$ The Lower Mainland Pharmacy Services (British Columbia) drug formulary currently lists 3 drugs in this class, all of which are subject to restrictions: ertapenem, imipenemcilastatin, and meropenem. For multidrug-resistant organisms where other agents are ineffective and cases in which the patient is intolerant to alternatives, imipenem-cilastatin is recommended as the first-line carbapenem, with meropenem to be used if imipenem-cilastatin is unsuitable, and ertapenem being reserved for patients undergoing treatment in an outpatient setting. Because of cost considerations, meropenem was used as the primary carbapenem at Abbotsford Regional Hospital during the period of this study.

A recent evaluation of carbapenem prescribing practices within 13 acute care sites serviced by the Lower Mainland Pharmacy Services ${ }^{5}$ revealed that use of carbapenems at Abbotsford Regional Hospital was higher than the regional mean when corrected for patient numbers. Given that this class of antibiotic should be reserved for severe cases and that the excessive and inappropriate use of carbapenems has been linked to plasmidmediated carbapenem resistance, ${ }^{2,6}$ it is important to understand how this drug class is being prescribed and to identify whether carbapenems are being overprescribed. This quality improvement review was undertaken to describe the patients receiving carbapenems at Abbotsford Regional Hospital in an effort to guide future interventions aimed at changing practice.
The primary objective of the study was to identify the indications for use of carbapenems and to assess the appropriateness of carbapenem orders written between May 1 and July 31, 2013. Secondary objectives were to identify the frequency of risk factors for multidrug-resistant organisms among patients who received carbapenems, to determine the proportion of patients with step-down to appropriate narrow-spectrum therapies, and to determine the number of patients who received carbapenems and subsequently tested positive for $C$. difficile toxin B or gramnegative multidrug-resistant organisms during the same admission.

\section{METHODS}

For this retrospective chart review, all patients who received at least one dose of meropenem, imipenem-cilastatin, or ertapenem between May 1 and July 31, 2013, were eligible for inclusion. The review was conducted at Abbotsford Regional Hospital, a 307-bed, university-affiliated community hospital. The protocol was submitted to the Fraser Health Research Ethics Board, which deemed the study exempt from the need for ethics approval.

Records for the included patients were divided among 5 research team members (including the authors of this article), who independently reviewed the details for each recorded visit and filled out a data collection form using a Microsoft InfoPath (Microsoft Corp, Redmond, Washington) template. Before reviewing these records, the 5 team members pilot-tested the data collection sheets using information for 3 excluded patients. The results of this pilot extraction were assessed by A.K. and D.M. together to ensure homogeneity of data collection. One team member (A.K.) collated all collected data into a Microsoft Excel (Microsoft Corp) spreadsheet.

The data were analyzed by 3 investigators (the authors of this article), who assessed the appropriateness of carbapenem use, according to criteria for appropriate, suboptimal, or inappropriate use. These criteria (see Box 1) were based on a previously published carbapenem assessment ${ }^{7}$ and incorporated additional input from 
This single copy is for your personal, non-commercial use only.

For permission to reprint multiple copies or to order presentation-ready copies for distribution, contact CJHP at cjhpedit@cshp.ca

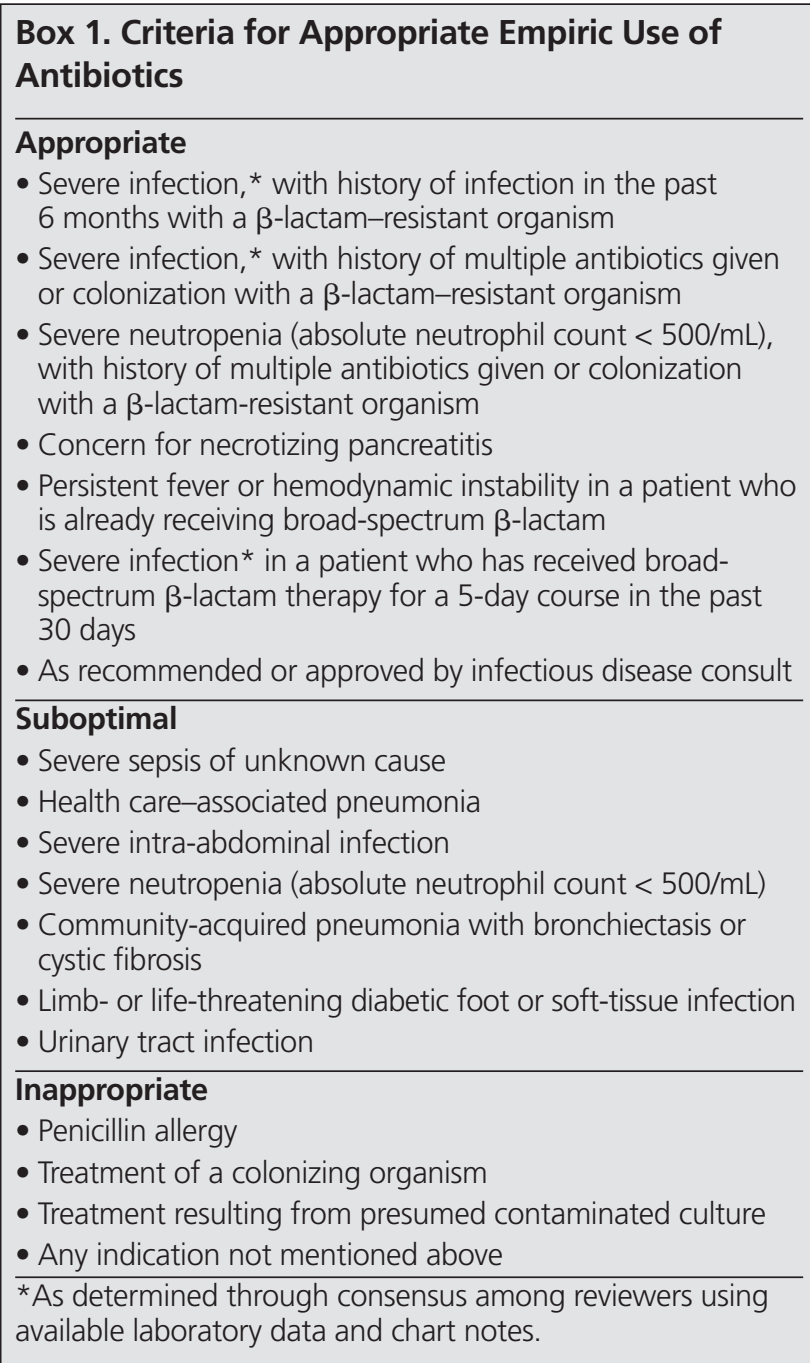

a local infectious disease specialist. Orders were deemed inappropriate if no obvious indication for carbapenem was found in the patient's record, suboptimal if alternative narrower-spectrum agents were available to treat the indication, and appropriate if no other therapeutic options existed. Each of the 3 authors reviewed all of the included cases to determine the appropriateness of carbapenem use. Conflicts in assessment were resolved on the basis of "majority rules".

\section{RESULTS}

\section{Baseline Characteristics}

A total of 299 eligible patients were identified through the pharmacy's computerized database. Of these, 200 were randomly chosen using a random number generator. Ultimately, data for 198 patients were used in the final analysis, as the detailed review indicated that 2 of the patients had received carbapenem in error. Table 1 describes the patients' baseline health characteristics and summarizes their antibiotic exposure in hospital before initiation
Table 1. Baseline Characteristics of Participants

\begin{tabular}{lc} 
Characteristic & $\begin{array}{c}\text { Mean } \pm \text { SD or } \\
\text { No. }(\%) \text { of Patients } \\
(\boldsymbol{n}=\mathbf{1 9 8})\end{array}$ \\
\hline Age (years) & $68 \pm 18.5$ \\
\hline Sex, male & $102(52)$ \\
\hline Medical history & $58(29)$ \\
Diabetes mellitus & $54(27)$ \\
Cancer & $45(23)$ \\
Chronic kidney failure & $31(16)$ \\
Recurrent urinary tract infection & $17(9)$ \\
COPD &
\end{tabular}

History of antimicrobial exposure in

hospital over past 3 months

Cephalosporins $32(16)$

$\beta$-Lactam and inhibitor combination $\quad 16 \quad$ (8)

Quinolones $16 \quad$ (8)

Macrolides $\quad 7 \quad$ (4)

Clindamycin $\quad 4 \quad$ (2)

Penicillins 3 (2)

Sulfa drugs 1 (1)

COPD = chronic obstructive pulmonary disease,

$\mathrm{SD}=$ standard deviation.

of carbapenem. Notably, 54 patients (27\%) had received some type of antibiotic during their admission before starting carbapenem therapy.

The carbapenem most commonly used during the study period was meropenem, which was used in 168 patients (85\%). The rates of use of ertapenem (16 patients) and imipenemcilastatin (14 patients) were similar to one another, but substantially lower than the rate for meropenem.

\section{Primary Outcome}

According to the aforementioned criteria (see Box 1), empiric use of carbapenems was assessed as appropriate in 41 (21\%) of the cases, suboptimal in 84 (42\%), and inappropriate in 73 (37\%) (Table 2). For most of the patients whose carbapenem therapy was deemed appropriate, the medication had been recommended by an infectious diseases physician or the patients had severe sepsis and a history of infection with extended-spectrum $\beta$-lactamase (ESBL)-producing organisms. Of the suboptimal carbapenem orders, the majority were for urinary tract infections or severe intra-abdominal infections in patients without a previous history of colonization with ESBL-producing organisms. Most of the patients whose carbapenem therapy was deemed inappropriate had nonspecific illnesses, where the patient was unwell with no obvious infectious process or focus of infection.

\section{Secondary Outcomes}

Table 3 summarizes the risk factors for multidrug-resistant organisms $s^{8}$ in the studied population. In this patient sample, $126(64 \%)$ had at least one risk factor for multidrug-resistant organisms, with therapy interpreted as appropriate for 35, suboptimal for 54, and inappropriate for 37 . Notably, the rationale 
This single copy is for your personal, non-commercial use only.

For permission to reprint multiple copies or to order presentation-ready copies for distribution, contact CJHP at cjhpedit@cshp.ca

Table 2. Assessment of Appropriateness of Carbapenem Therapy

\begin{tabular}{|c|c|c|}
\hline Characteristic & No. (\%) o & f Patients* \\
\hline Appropriate & 41 & (21) \\
\hline $\begin{array}{l}\text { Recommendation from infectious } \\
\text { diseases physician }\end{array}$ & 27 & (66) \\
\hline $\begin{array}{l}\text { Sepsis with colonization by ESBL- } \\
\text { producing organism }\end{array}$ & 10 & $(24)$ \\
\hline $\begin{array}{l}\text { Persistent fever or hemodynamic } \\
\text { instability in patient already receiving } \\
\text { broad-spectrum } \beta \text {-lactam }\end{array}$ & 2 & (5) \\
\hline Necrotizing pancreatitis & 2 & (5) \\
\hline Suboptimal & 84 & (42) \\
\hline Urinary tract infection & 44 & $(52)$ \\
\hline Severe intra-abdominal infection & 11 & (13) \\
\hline Severe sepsis & 9 & $(11)$ \\
\hline Neutropenia & 7 & (8) \\
\hline Hospital-acquired pneumonia & 6 & (7) \\
\hline Health care-associated pneumonia & 2 & (2) \\
\hline Diabetic foot & 2 & (2) \\
\hline Limb-threatening soft-tissue infection & 2 & (2) \\
\hline Nursing home-associated pneumonia & 1 & (1) \\
\hline Inappropriate & 73 & (37) \\
\hline Nonspecific illness & 45 & (62) \\
\hline Community-acquired pneumonia & 14 & (19) \\
\hline Ischemic bowel & 2 & (3) \\
\hline Preoperative therapy & 1 & (1) \\
\hline Gastritis & 1 & (1) \\
\hline Aspiration pneumonia & 1 & (1) \\
\hline Appendicitis & 1 & (1) \\
\hline Pancreatitis & 1 & (1) \\
\hline Appendiceal abscess & 1 & (1) \\
\hline Necrotizing fasciitis & 1 & (1) \\
\hline Colitis & 1 & (1) \\
\hline Peritonitis & 1 & (1) \\
\hline COPD exacerbation & 1 & (1) \\
\hline Abdominal pain & 1 & (1) \\
\hline Diverticulitis & 1 & (1) \\
\hline
\end{tabular}

$\mathrm{C}=\mathrm{COPD}=$ chronic obstructive pulmonary disease,

$\mathrm{ESBL}=$ extended-spectrum $\beta$-lactamase.

*For each of the 3 category subtotals, the percentage is based on the total sample $(n=198)$. Within each category, the percentages are based on the number in that category.

\section{Table 3. Risk Factors for Multidrug-Resistant Organisms}

\begin{tabular}{lrr} 
Risk Factor & $\begin{array}{c}\text { No. (\%) of Patients } \\
(\boldsymbol{n}=\mathbf{1 9 8})\end{array}$ \\
\hline $\begin{array}{l}\text { History of antimicrobial exposure in } \\
\text { hospital over past 3 months }\end{array}$ & $70(35)$ \\
Immunosuppression & $50(25)$ \\
Catheter in place & $45(23)$ \\
Hospital stay > 5 days & $29(15)$ \\
Admission to nursing home & $23(12)$ \\
Colonization with ESBL-producing & 16 & $(8)$ \\
organism & & \\
Critical illness & 11 & $(6)$ \\
At least 1 risk factor & 126 & $(64)$ \\
At least 2 risk factor & 65 & $(33)$ \\
\hline
\end{tabular}

$\mathrm{ESBL}=$ extended-spectrum $\beta$-lactamase. for choice of carbapenem versus other antibiotics was documented in only 68 (34\%) of all 198 cases. Logistic regression revealed that a hospital stay of more than 5 days $(p=0.041)$ and colonization with ESBL-producing organisms $(p<0.001)$ were statistically significant predictors of appropriate carbapenem use. Sixty-two (31\%) of the patients had step-down from the initial carbapenem to a narrower-spectrum agent. Eight of the patients (4\%) acquired C. difficile subsequent to carbapenem exposure, and $18(9 \%)$ became infected with multidrug-resistant organisms (excluding methicillin-resistant Staphylococcus aureus).

Table 4 describes the sites of infection among the patients who received carbapenems. Documentation of the specific site of infection was lacking for 53 (27\%) cases, and an additional $53(27 \%)$ cases had documentation of a genitourinary source. Of the 375 samples sent for culture and sensitivity testing, 255 (68\%) were negative. The most common organism isolated was Escherichia coli (47 samples [13\%]). Only 7 samples grew P. aeruginosa.

\section{DISCUSSION}

This review showed that carbapenems prescribed at Abbotsford Regional Hospital were directed at a wide range of infections. Although much of the prescribing was within Health Canada's approved indications, ${ }^{9} 73$ (37\%) of the carbapenem orders were deemed inappropriate, and 84 (42\%) were suboptimal, which indicates that there is potential for use of narrower-spectrum agents.

Weston and others ${ }^{7}$ conducted a retrospective study at 4 Veterans Affairs hospitals in the United States using similar appropriateness criteria for the assessment of carbapenem orders. They found that prescribing of carbapenem was appropriate for about $70 \%$ of cases in the health system, a result that differs significantly from the result presented here. ${ }^{7}$ Unlike the protocols in place at the site studied by Weston and others, the Abbotsford Regional Hospital protocol did not require approval from an infectious diseases physician before a second carbapenem dose, which thus avoided any practical disincentives to ordering carbapenems without thorough consideration of alternatives. In addition, the formulary restriction criteria for carbapenems at Abbotsford Regional Hospital were relatively new at the time of data collection and difficult for pharmacy staff to enforce. For other restricted medications, formulary policies can be enforced by withholding medications until pharmacy approval has been obtained; however, the adverse implications of possible delays in therapy preclude this tactic for enforcing restriction criteria for carbapenems. The majority of the inappropriate carbapenem orders were defined as such because no clear indication or obvious infectious cause had been documented by prescribers.

Relative to other acute care sites in the Lower Mainland, the carbapenem prescribing pattern at Abbotsford Regional Hospital could be due to the region's cultural diversity. A greater proportion of individuals in Abbotsford are of South Asian descent relative 
This single copy is for your personal, non-commercial use only.

For permission to reprint multiple copies or to order presentation-ready copies for distribution, contact CJHP at cjhpedit@cshp.ca

\section{Table 4. Sites of Infection for Patients Treated with Carbapenems}

Suitability of Therapy; No. (\%) of Cases

\begin{tabular}{lccccc}
\cline { 2 - 6 } Site of Infection & Appropriate & Suboptimal & Inappropriate & Overall $(\boldsymbol{n}=198)$ \\
\hline Central nervous system & 0 & 0 & 1 & $1<1)$ \\
Respiratory system & 6 & 11 & 16 & 33 & $(17)$ \\
Gastrointestinal system & 4 & 10 & 12 & 26 & $(13)$ \\
Genitourinary system & 13 & 37 & 3 & 53 & $(27)$ \\
Skin, musculoskeletal, or vascular system & 8 & 3 & 2 & 13 & $(7)$ \\
Blood & 4 & 8 & 7 & 19 & $(10)$ \\
Unknown & 6 & 15 & 32 & 53 & $(27)$ \\
\hline
\end{tabular}

to Vancouver (16\% versus $10 \%$, respectively). ${ }^{10}$ Tangden and others ${ }^{11}$ showed that travellers to India were at higher risk of colonization with ESBL-producing organisms than travellers to other regions. Clinicians at Abbotsford Regional Hospital are likely to be more wary of ESBL colonization because of the demographic characteristics of their patient populations, and hence may be more likely to use carbapenems. However, colonization with an ESBL-producing organism was confirmed in only 16 $(8 \%)$ of the patients receiving carbapenems in this study.

About one-third of patients receiving carbapenems had stepdown to a narrower-spectrum agent. This relatively low rate may be due to absence of growth on culture of the majority of specimens, providing insufficient information to guide therapy. Although "most responsible physician" was not a prespecified outcome, the majority of patients without step-down from carbapenem therapy were in the care of primary care physicians, who may not have the resources to monitor patients after a change in therapy in the absence of appropriate guiding culture results. Nevertheless, antimicrobial step-down as soon as culture and sensitivity results are available is integral to antibiotic stewardship, to minimize resistance rates and other complications of suboptimal and inappropriate antibiotic use. ${ }^{3,12}$

This study is the first audit of carbapenem prescribing at Abbotsford Regional Hospital following the implementation of restrictions on this drug class. Because the majority of patients receiving carbapenem during the study period were assessed, these findings are likely representative of the local carbapenem prescribing patterns. Another strength of the project was the use of criteria similar to those used in previous studies to assess carbapenem orders; as such, the results presented here can be compared with those in the literature.

The study had several limitations. Because the study was a retrospective chart review, the information specified in the data collection form was not always available: in several cases, data for certain patient characteristics were insufficient for assessment, including severity of patient illness, outpatient antibiotic use, recent travel history, and medical history before admission. Inadequate documentation may bias prescribing patterns by making them appear worse than reality. In addition, the hindsight bias associated with retrospective studies, whereby assessments made after the fact may not accurately reflect the acuity of the patient's condition at the time of prescription, may have led to further underestimation of the rate of appropriate ordering. Finally, it is important to recognize the potential for seasonal bias, given that the study period was May to July. An epidemiological study conducted in the United States showed that the incidence of and mortality rate associated with sepsis were higher during the winter months. ${ }^{13}$

Unfortunately, even if these limitations led to underestimation of rates of appropriate carbapenem use, the ample room for improvement combined with the relatively high usage at Abbotsford Regional Hospital compels corrective actions. Future interventions may centre on the requirement for preprinted orders, with standards for documentation of clinical rationale before dispensing of the first dose of a carbapenem and incorporation of advanced technology such as computerized physician order entry to further support the prescribing of carbapenems. To increase step-down from carbapenems to narrower-spectrum therapy in the absence of microbiology results, clinicians should be reminded of existing prescribing guidelines for empiric therapy to inform any planned changes in therapy. Continued monitoring of carbapenem prescribing patterns and reassessment of the appropriateness of orders according to standard criteria should be conducted regularly.

\section{CONCLUSION}

At Abbotsford Regional Hospital, carbapenems were prescribed for a wide variety of indications. During the study period, only $21 \%$ of carbapenem orders were considered appropriate, according to prespecified, literature-based criteria. These findings indicate that approximately $80 \%$ of patients with carbapenem orders at Abbotsford Regional Hospital could benefit from the use of a narrower-spectrum agent.

References

1. Brink AJ, Feldman C, Grolman DC, Muckart D, Pretorius J, Richards GA, et al. Appropriate use of the carbapenems. S Afr Med J. 2004;94(10 Pt 2):857-61.

2. Nordmann P, Naas T, Poirel L. Global spread of carbapenemase-producing Enterobacteriaceae. Emerg Infect Dis. 2011;17(10):1791-8.

3. Spellberg B, Guidos R, Gilbert D, Bradley J, Boucher HW, Scheld WM, et al.; Infectious Diseases Society of America. The epidemic of antibiotic- 
resistant infections: a call to action for the medical community from the Infectious Diseases Society of America. Clin Infect Dis. 2008;46(2):155-64.

4. McLaughlin M, Advincula MR, Malczynski M, Qi C, Bolon M, Scheetz M. Correlations of antibiotic use and carbapenem resistance in Enterobacteriaceae. Antimicrob Agents Chemother. 2013;57(10):5131-3.

5. Lower Mainland consolidation initiative quick facts. Vancouver (BC): Vancouver Coastal Health; 2011 Nov. p. A11.

6. Jary F, Kaiser JD, Henon T, Leroy L, Patry I, Blasco G, et al. Appropriate use of carbapenems in the Besançon University Hospital. Med Mal Infect. 2012;42(10):510-6.

7. Weston G, Jacob JT, Ray S, Varkey J, Gaynes RP. A multicenter study measuring appropriateness of carbapenem use. Infect Control Hosp Epidemiol. 2013;34(12):1324-6.

8. Trecarichi EM, Cauda R, Tumbarello M. Detecting risk and predicting patient mortality in patients with extended-spectrum $\beta$-lactamase-producing Enterobacteriaceae bloodstream infections. Future Microbiol. 2012;7(10): 1173-89.

9. Meropenem [product monograph]. In: $e$-CPS [electronic database]. Ottawa (ON): Canadian Pharmacists Association; 2012. Subscription required to access content.

10. Population estimates and projections: Visible minority population, by census metropolitan areas (2006 Census) (Abbotsford-Mission, Vancouver, Victoria). Ottawa (ON): Statistics Canada; 2006 [cited 2014 Sep 12]. Available from: www.statcan.gc.ca/tables-tableaux/sum-som/101/cst01/ demo53g-eng.htm

11. Tängdén T, Cars O, Melhus A, Löwdin E. Foreign travel is a major risk factor for colonization with Escherichia coli producing CTX-M-type extendedspectrum beta-lactamases: a prospective study with Swedish volunteers. Antimicrob Agents Chemother. 2010;54(9):3564-8.
12. Mertz D, Koller M, Haller P, Lampert ML, Plagge H, Hug B, et al. Outcomes of early switching from intravenous to oral antibiotics on medical wards. J Antimicrob Chemother. 2009;64(1):188-99.

13. Danai PA, Sinha S, Moss M, Haber MJ, Martin GS. Seasonal variation in the epidemiology of sepsis. Crit Care Med. 2007;35(2):410-5.

Jesse Janssen, BSc, MD, is with the Faculty of Medicine, McGill University, Montréal, Quebec.

Angus Kinkade, BSC(Pharm), ACPR, PharmD, MSc, BCPS, is with Lower Mainland Pharmacy Services, Vancouver, British Columbia.

Damen Man, BSc(Pharm), ACPR, PharmD, is with Lower Mainland Pharmacy Services, Vancouver, British Columbia.

Competing interests: None declared.

\section{Address correspondence to:}

Dr Damen Man

Pharmacy Department

Abbotsford Regional Hospital

32900 Marshall Road

Abbotsford BC V2S OC2

e-mail: Damen.Man@fraserhealth.ca

Acknowledgements: The authors thank Hyo-Jung Moon and Davie Lam for their contributions to data collection for this project.

Funding: None received.

\section{Canadian Society of Hospital Pharmacists Société canadienne des pharmaciens d'hôpitaux Membership Year 2015/2016 (July 1, 2015 - June 30, 2016)}

Are you a Member or Supporter of the Canadian Society of Hospital Pharmacists?

CSHP has celebrated 67 years of inspiration and leadership among a growing network of over 3000 pharmacy colleagues. When you join CSHP, you have access to resources and opportunities that will enhance your career and help influence healthcare in Canada. Thank you for your participation

in CSHP 2015, an initiative challenging all pharmacists to improve patient medication outcomes and safety through advancing pharmacy practice excellence by the year 2015.

\section{Renew or join today!}

Membership Enquiries: Please contact Membership Services Tel.: (613) 736-9733 Ext. 222 | Fax: (613) 736-5660 |Email: membershipservices@cshp.ca www.cshp.ca 